\title{
Sustained multiple organ ischaemia after transarterial chemoembolization with drug-eluting beads for hepatocellular carcinoma
}

\author{
YOUNG WOON KIM ${ }^{1}$, JUNG HYUN KWON ${ }^{1}$, SOON WOO NAM ${ }^{1}$, JEONG WON JANG ${ }^{2}$, \\ HYUN SUK JUNG ${ }^{3}$, YU RI SHIN ${ }^{3}$, EUN SU PARK ${ }^{4}$ and DONG JAE SHIM ${ }^{3}$
}

\author{
${ }^{1}$ Department of Internal Medicine, Division of Hepatology, Incheon St. Mary's Hospital, The Catholic University of \\ Korea, Incheon 21431; ${ }^{2}$ Department of Internal Medicine, Division of Hepatology, Seoul St. Mary's Hospital, \\ The Catholic University of Korea, Seoul 06591; Departments of ${ }^{3}$ Radiology and ${ }^{4}$ Pathology, Incheon St. Mary's Hospital, \\ The Catholic University of Korea, Incheon 21431, Republic of Korea
}

Received August 9, 2017; Accepted October 26, 2017

DOI: $10.3892 / \mathrm{etm} .2017 .5540$

\begin{abstract}
Transarterial chemoembolization (TACE) with drug-eluting beads (DC beads) may enhance drug delivery to tumours and reduce systemic toxicity. TACE with DC beads leads to significantly fewer serious side-effects compared with conventional TACE. A 66-year-old man with hepatocellular carcinoma (HCC) complained of continuous abdominal pain 1 month after TACE with DC beads. At the time of TACE, angiography revealed severe stenosis of both hepatic arteries. The diagnostic work up on admission suggested severe bile duct injury with regional bile duct dilatation, segmental liver and spleen infarction, necrotizing pancreatitis, as well as gastric and duodenal ulcers. The pathology specimens of the duodenum contained DC beads that had passed through small vessels in the connective tissue. The patient's condition appeared to improve after 2 weeks of antibiotic treatment and supportive care, but new multifocal liver and spleen infarction subsequently developed. After 2 months, he was well enough to be discharged. His HCC partially responded to the TACE with DC beads but eventually progressed and he died after 11 months. The present case report highlights unexpected ongoing multiple organ ischaemia in a 66-year-old man treated for HCC using TACE with DC beads. The use of TACE with DC beads should be carefully considered in patients with vascular strictures or aberrant blood supply.
\end{abstract}

Correspondence to: Dr Jung Hyun Kwon, Department of Internal Medicine, Division of Hepatology, Incheon St. Mary's Hospital, The Catholic University of Korea, 56 Dongsu-ro, Bupyeong-gu, Incheon 21431, Republic of Korea

E-mail: wdoctorkwon@catholic.ac.kr

Key words: hepatocellular carcinoma, transarterial chemoembolization, doxorubicin-loaded beads, ischemia

\section{Introduction}

Hepatocellular carcinoma (HCC) is a common type of cancer that is usually diagnosed at an advanced stage. Transarterial chemoembolization (TACE) is widely used to treat HCC patients who cannot be given curative treatments $(1,2)$. The TACE protocol includes intra-arterial chemotherapy with lipiodol and chemotherapeutic agents, followed by selective vascular embolization with gelfoam. A study comparing TACE with best supportive care found a distinct survival benefit with TACE $(1,2)$. In addition, the transarterial chemolipidolization (TACL) protocol includes transarterial infusion of chemotherapeutic agents in a mixture of lipiodol without gelfoam embolization (3-5). TACL may be performed multiple times with minimization of vascular injury and also provided the benefit of downsizing of HCC (3-5). However, TACE causes a range of minor and major complications, including acute cholecystitis, ischaemic hepatitis, pulmonary thromboembolism, liver abscess, ischaemic biliary strictures and, less frequently, pancreatic damage. In particular, TACE may cause injury to the hepatic artery, leading to arterial spasms and inflammatory constriction, and occlusion or dissection in severe cases (6-8). Drug-eluting beads (DC beads) loaded with chemotherapeutic agents have been developed, which facilitate slow drug release upon injection. This may increase the intensity and duration of tumour ischaemia as a result of enhancing drug delivery to the tumour $(9,10)$. Several studies have demonstrated that TACE with DC beads has fewer systemic side effects and less toxicity than conventional TACE (10-12). However, the present case reports on the case of a patient who unexpectedly experienced sustained multiple-organ ischaemia including pancreatitis, peptic ulcer and multiple liver-spleen infarctions after TACE with DC beads. This case is unusual but suggests the requirement for careful use of TACE with DC beads and early recognition of ischaemic complications.

\section{Case report}

A 66-year-old man with HCC was admitted to the Department of Internal Medicine, Incheon St. Mary's Hospital, (Incheon, 
A

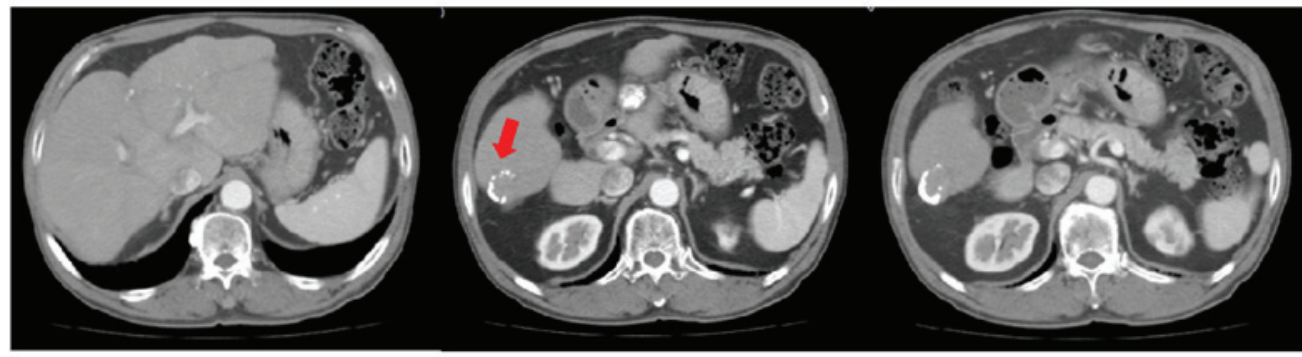

B

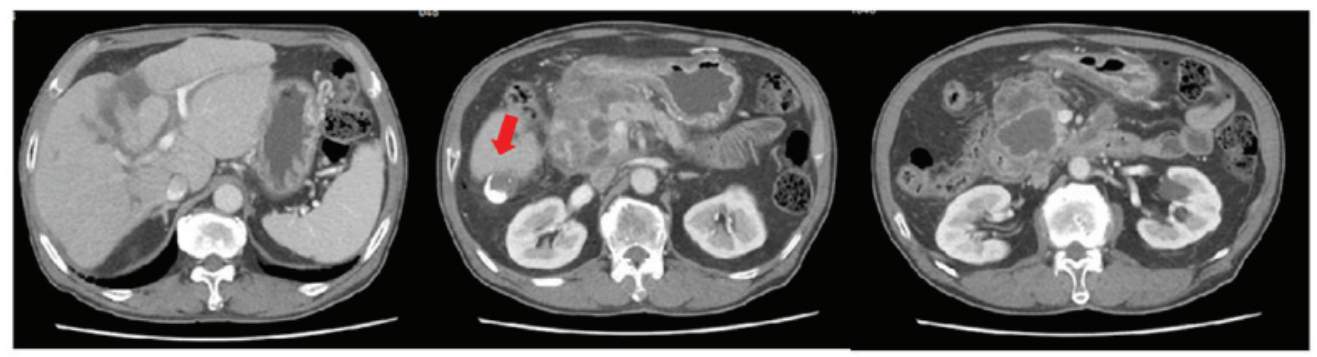

C

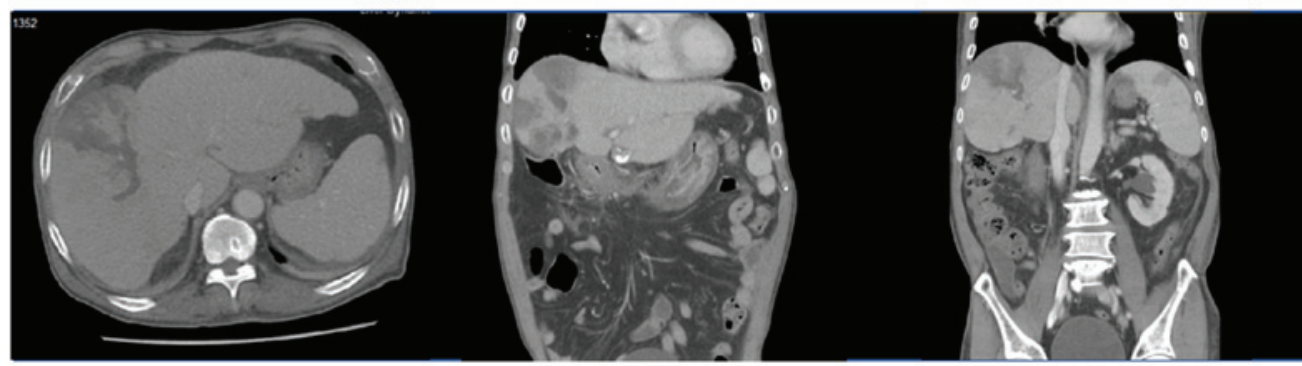

Figure 1. (A) Liver dynamic CT performed 2 days before the TACE with DC beads showing a viable remnant tumour (segment 6, arrow). (B) Liver dynamic CT performed on admission due to abdominal pain revealed multi-locular fluid accumulation with enhanced walls in the peripancreatic space and marked irregular right intrahepatic duct dilatation. However, imaging indicated increased necrosis $(3 \mathrm{~cm})$ in the lipiodol-laden hepatocellular carcinoma at segment 6 (C) Liver dynamic CT re-performed for fever and abdominal pain after initial improvement (44 days after the TACE with DC beads) revealing newly developed multifocal perfusion defects, suggesting liver and splenic infarction. The peri-pancreatic fluid collection was decreased. DC beads, drug-eluting beads; $\mathrm{CT}$, computed tomography.

South Korea) due to diffuse abdominal pain that began 1 month after TACE with DC beads. Six years previously, at the same hospital the patient had been diagnosed with a single $2.6-\mathrm{cm}$ HCC lesion in segment 7 (Barcelona Clinic Liver Cancer Class A, Okuda Stage 1, Eastern Cooperative Oncology Group Status 1). The patient refused surgical resection and was treated with TACL using adriamycin $(50 \mathrm{mg})$. Of note, the protocol at our institution does not use conventional TACE but TACL. The patient had been treated with 19 cycles of on-demand TACL over 6 years, whenever a new nodule developed. No distant metastasis was observed over this time period. The initial HCC necrotized following 4 cycles of TACL. Subsequently, a 2-cm HCC in segment 4 developed and was treated with 10 cycles of TACL. After 18 months of complete remission, a 2.2-cm HCC in segment 3 developed and necrotized for 3 cycles of TACL. After 10 months, a 2.4-cm HCC in segment 6 newly developed and was treated two times with TACL. However, a viable remnant of the last target lesion was still present (Fig. 1). Finally, the patient was treated by TACE with DC beads to reduce the frequency of TACL at 1 month prior to the current admission (Fig. 1A). The patient had possessed an intact hepatic artery at the first TACL procedure, but narrowing and damage of the artery progressed with multiple TACLs (Fig. 2). The current angiogram revealed that both hepatic arteries were narrow, and thin aberrant collateral vessels to the tumour were observed
(Fig. 2B). To prepare DC beads, $50 \mathrm{mg}$ doxorubicin was loaded in a 2-ml vial containing 100-300 $\mu \mathrm{m}$ beads (Biocompatibles, Farnham, UK) with nonionic contrast media (Imeron 300; Bracco-Altana, Konstanz, Germany). After saturation of the DC beads, the radiologist started to infuse them at a rate of $1 \mathrm{ml} / \mathrm{min}$. However, he unexpectedly encountered difficulties infusing the DC beads into the right hepatic artery, and detected a small backward flow of the DC beads. The infusion of beads was immediately stopped and no additional stained tumour was seen on hepatic angiography.

On the current admission, the patient complained of continuous abdominal discomfort, particularly in the periumbilical area. Liver dynamic computed tomography (CT) confirmed severe bile duct injury, liver infarction in the right hepatic lobe and newly developed necrotizing pancreatitis (Fig. 1B). The targeted HCC nodule exhibited an increased area of necrosis compared with that seen 1 month earlier (Fig. 1B). Gastrofibroscopy (GFS) revealed multiple active ulcers on the antrum and body of the stomach and oedematous, ulcerative lesions on the proximal second portion of the duodenum (Fig. 3). On the pathology specimen of the duodenum, DC-beads that had passed through small vessels in the duodenal connective tissue were observed (Fig. 4).

After admission, the patient's symptoms were treated conservatively with broad-spectrum antibiotics, hydration, 

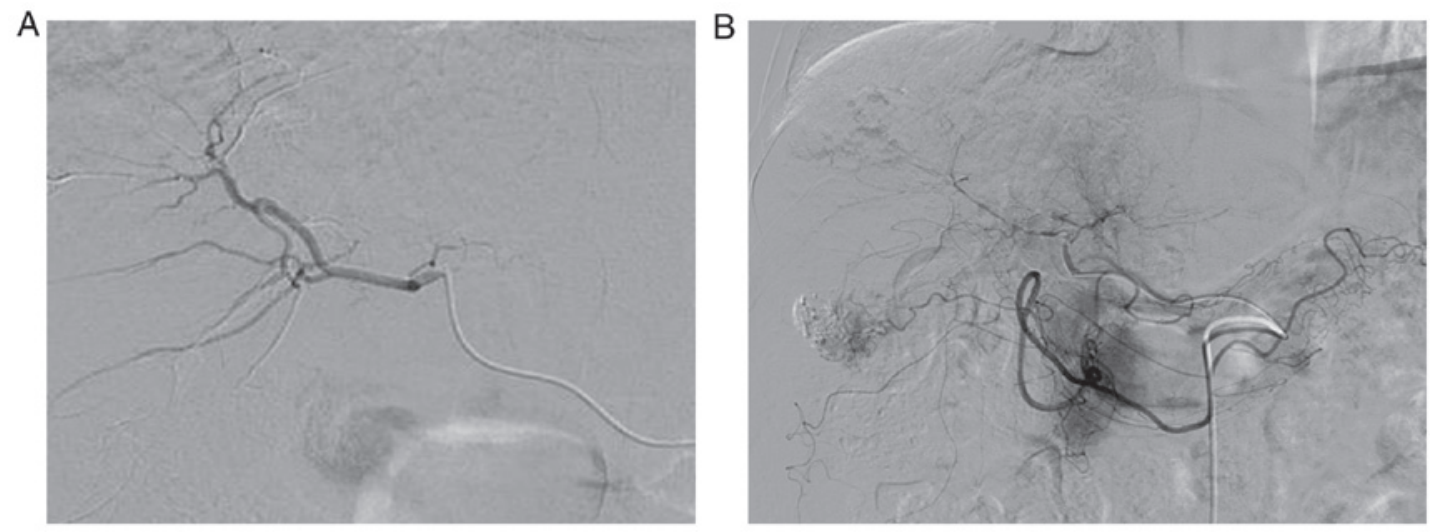

Figure 2. Angiograms revealing (A) the intact hepatic artery at the first transarterial chemolipidolization procedures and (B) marked narrowing of the right hepatic artery at the time of trans-arterial chemo-embolization with DC beads. The narrowing made infusion of DC beads into the right hepatic artery difficult and produced a backward flow that required immediate cessation of the infusion. DC beads, drug-eluting beads.
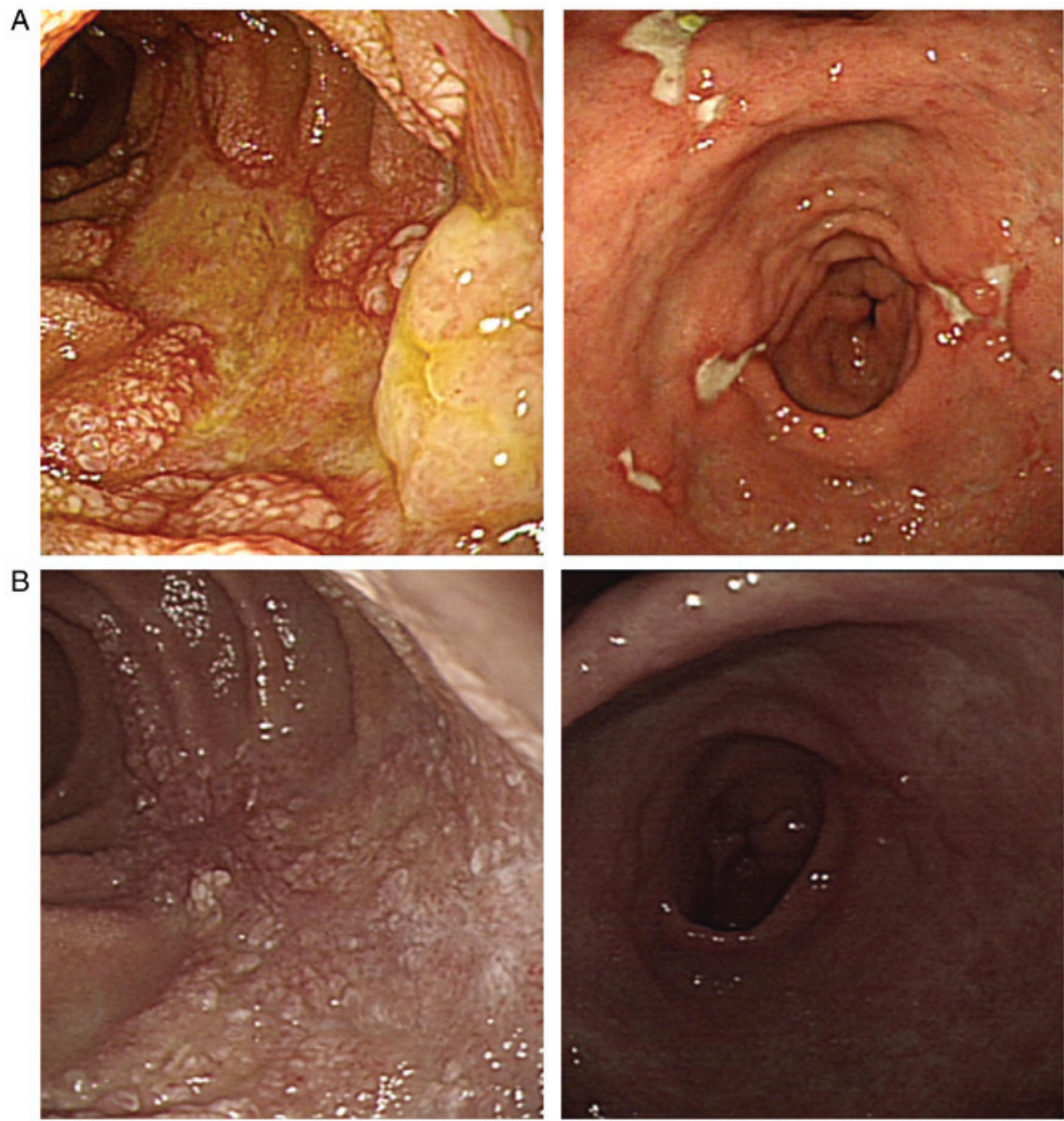

Figure 3. (A) Gastrofibroscopy revealing an oedematous, ulcerative lesion on the proximal second portion of the duodenum and multiple active ulcers on the angle and body of the stomach at 30 days after TACE with DC beads. (B) Duodenal and gastric ulcers had healed well at 63 days after TACE with DC-beads. DC beads, drug-eluting beads; TACE, trans-arterial chemo-embolization.

pain control, anti-ulcer medication and a proteinase inhibitor for necrotizing pancreatitis. His condition had improved 2 weeks after admission. However, at this time-point (44 days after the TACE with DC beads), he developed an elevated temperature of $38.7^{\circ} \mathrm{C}$ and marked elevations of aspartate aminotransferase, alanine aminotransferase, $\mathrm{C}$-reactive protein and total bilirubin (Table I). Follow-up abdominal CT images revealed a newly developed multifocal liver infarction in the right hepatic lobe and a newly developed partial infarction in the spleen (Fig. 1C). Finally, 30 days after admission (2 months after the TACE with DC beads), his symptoms and laboratory results improved (Table I). On the subsequent GFS examination, the gastric ulcer and duodenal ulcer exhibited signs of healing (Fig. 3B) and the patient was discharged.

After recovering from the multiple hepatic infarcts and ongoing ischaemic changes, the patient had no additional DC 
Table I. Laboratory results at various time-points after TACE with DC beads.

\begin{tabular}{|c|c|c|c|c|}
\hline Parameter & Prior to TACE with beads & Day 30 (Admission) & Day 44 (Fever) & Day 64 (Discharge) \\
\hline \multicolumn{5}{|l|}{ Complete blood cell count } \\
\hline Hemoglobin (g/dl) & 12.3 & 11.0 & 8.9 & 10.3 \\
\hline WBC count $\left(10^{3} / \mu 1\right)$ & 10,970 & 12,010 & 9,490 & 5,830 \\
\hline Platelet count $\left(10^{3} / \mu 1\right)$ & 197 & 345 & 59 & 274 \\
\hline \multicolumn{5}{|l|}{ Blood chemistry } \\
\hline BUN (mg/dl) & 19.1 & 30.9 & 9.8 & 15.1 \\
\hline Creatinine (mg/dl) & 0.8 & 0.9 & 0.7 & 0.6 \\
\hline Total protein $(\mathrm{g} / \mathrm{dl})$ & 6.9 & 6.4 & 4.7 & 6.5 \\
\hline Albumin $(\mathrm{g} / \mathrm{dl})$ & 3.8 & 3.1 & 2.4 & 2.9 \\
\hline $\operatorname{AST}(\mathrm{U} / \mathrm{l})$ & 32 & 82 & 1305 & 38 \\
\hline ALT (U/l) & 28 & 42 & 496 & 18 \\
\hline Total bilirubin (mg/dl) & 0.5 & 1.2 & 3.5 & 2.0 \\
\hline Direct bilirubin (mg/dl) & 0.1 & 0.5 & 2.3 & 0.9 \\
\hline r-GTP (U/1) & 69 & 153 & 439 & 459 \\
\hline ALP (U/1) & 133 & 280 & 603 & 475 \\
\hline Amylase (IU/l) & 56 & 219 & 80 & 64 \\
\hline $\mathrm{CRP}(\mathrm{mg} / \mathrm{l})$ & 4.43 & 133.92 & 133.97 & 24.66 \\
\hline \multicolumn{5}{|l|}{ Coagulation test } \\
\hline Prothrombin time (INR/\%) & 1.02 & 1.06 & 1.31 & 1.20 \\
\hline \multicolumn{5}{|l|}{ Tumor markers } \\
\hline $\operatorname{AFP}(\mathrm{ng} / \mathrm{ml})$ & 190.76 & 22.43 & & \\
\hline PIVKA II & 367 & 70 & & \\
\hline
\end{tabular}

BUN, blood urea nitrogen; AST, aspartate aminotransferase; ALT, alanine aminotransferase; INR, international normalized ratio; DC beads, drug-eluting beads; TACE, trans-arterial chemo-embolization; AFP, $\alpha$-fetoprotein; PIVKA II, acarboxy prothrombin.

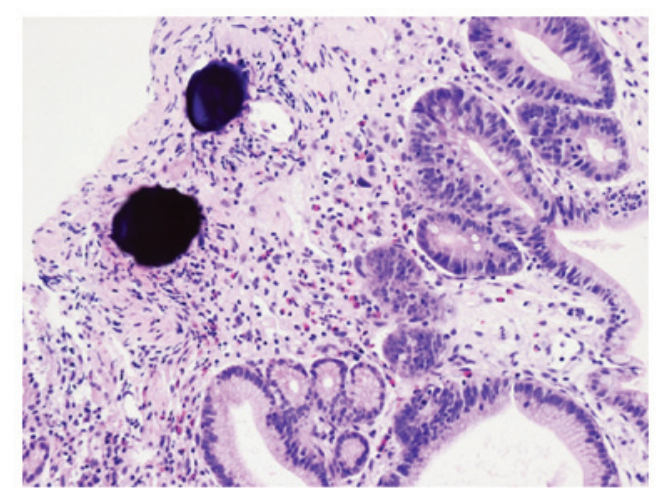

Figure 4. Haematoxylin-stained DC beads embedded in duodenal connective tissue and surrounded by lymphocytes 30 days after TACE with DC beads (magnification, x200). DC beads, drug-eluting beads. TACE, transarterial chemoembolization.

bead-associated multi-organ complications until he died due to the progression of HCC at 11 months after TACE with DC beads.

\section{Discussion}

The present case report showed a severe case of ischaemic inflammation of multiple organs after TACE with DC beads. A European multicentre randomized study reported that TACE with DC beads is more effective than conventional TACE in terms of disease control and tolerability (13). The use of DC beads allowing the slow release of chemotherapy over time has been correlated with fewer systemic complications, including nausea, vomiting, asthenia and alopecia compared with conventional TACE $(11,13)$. In the case of TACE with DC beads, severe adverse events including hepatic insufficiency, infarction or hepatic abscess, and tumour rupture may occur, but are associated with an excellent tumour response. Such complications are rare, with a reported incidence of 3-4\% and 30-day mortality rates of $1-4 \%(14,15)$, and usually occur within 30 days of the procedure (16). A previous study reported that TACE with DC beads was effective for refractory HCC compared with conventional TACE (17). A complete response was observed in $40 \%$ of the tumours and a partial response was observed in $60 \%$ of the tumours. TACE with DC beads elicited a better response than conventional TACE, particularly when the tumours were small and exhibited a delayed enhancement pattern upon angiography. In the present case report, the patient's HCC nodule was small.

Several studies have reported hepatic loco-regional toxicities of TACE with DC beads (18-22). The incidence of biliary injury and intrahepatic biloma were significantly higher following TACE with DC beads (18). In the present case report, the patient developed not only bile duct injury and liver infarction, but also spleen infarction and ischaemic duodenal ulcers over several months. Known risk factors associated with ischaemic organ damage after conventional TACE are non-selective 
embolization, the number of procedures and the volume of embolic material $(22,23)$. In particular, the baseline prothrombine value and the dose of chemotherapy have been correlated with the occurrence of hepatic loco-regional complications after TACE with DC beads (18). In the present case report, the vascular stricture resulting from numerous previous TACE treatments may have contributed to the multi-organ ischemia by causing a reflux of DC beads into the gastroduodenal, right gastric, right gastroepiploic, left gastric or splenic branches of the celiac trunk due to the stenosis of the hepatic artery. The beads displayed in the pathology sample of duodenal tissue suggested such a regurgitation of the DC beads. An animal study in Yucatan pigs demonstrated an increased retention and sustained release of doxorubicin after embolization with loaded DC beads for 28 until up to 90 days after liver embolization (24). Thus, ischaemic damage associated with the reflux of DC-beads may continue for $>3$ months and is more severe than that produced by conventional TACE $(24,25)$. To prevent this complication, it is important to reduce the backflow of embolic material by placing the catheter tip as close as possible to the distal branches of the hepatic artery as possible, and then carefully injecting the embolic material to avoid regurgitation. Particularly in patients with vessel stricture following previous repeated conventional TACE, the selection of TACE with DC beads as a treatment option should be made with caution.

In conclusion, TACE with DC beads should only be considered with caution, particularly in patients with stenosis of the hepatic artery, and a super-selective procedure is essential to avoid the embolization of DC beads in unintended organs. In addition, even considering its expected favourable antitumour effect, clinicians should be aware of the potential risk of an unusual fatal course after TACE with DC beads.

\section{References}

1. Lo CM, Ngan H, Tso WK, Liu CL, Lam CM, Poon RT, Fan ST and Wong J: Randomized controlled trial of transarterial lipiodol chemoembolization for unresectable hepatocellular carcinoma. Hepatology 35: 1164-1171, 2002.

2. Llovet JM, Real MI, Montaña X, Planas R, Coll S, Aponte J, Ayuso C, Sala M, Muchart J, Solà R, et al: Arterial embolisation or chemoembolisation versus symptomatic treatment in patients with unresectable hepatocellular carcinoma: A randomised controlled trial. Lancet 359: 1734-1739, 2002.

3. Jang JW, Park YM, Bae SH, Choi JY, Yoon SK, Chang UI, Nam SW and Kim BS: Therapeutic efficacy of multimodal combination therapy using transcatheter arterial infusion of epirubicin and cisplatin, systemic infusion of 5-fluorouracil, and additional percutaneous ethanol injection for unresectable hepatocellular carcinoma. Cancer Chemother Pharmacol 54: 415-420, 2004.

4. Jang JW, Bae SH, Choi JY, Oh HJ, Kim MS, Lee SY, Kim CW, Chang UI, Nam SW, Cha SB, et al: A combination therapy with transarterial chemo-lipiodolization and systemic chemo-infusion for large extensive hepatocellular carcinoma invading portal vein in comparison with conservative management. Cancer Chemother Pharmacol 59: 9-15, 2007.

5. Jang JW, You CR, Kim CW, Bae SH, Yoon SK, Yoo YK, Kim DG and Choi JY: Benefit of downsizing hepatocellular carcinoma in a liver transplant population. Aliment Pharmacol Ther 31: 415-423, 2010.

6. Maeda N, Osuga K, Mikami K, Higashihara H, Onishi H, Nakaya Y, Tatsumi M, Hori M, Kim T, Tomoda K, et al Angiographic evaluation of hepatic arterial damage after transarterial chemoembolization for hepatocellular carcinoma. Radiat Med 26: 206-212, 2008.

7. Sakamoto I, Aso N, Nagaoki K, Matsuoka Y, Uetani M, Ashizawa K, Iwanaga S, Mori M, Morikawa M, Fukuda T, et al: Complications associated with transcatheter arterial embolization for hepatic tumors. Radiographics 18: 605-619, 1998.
8. Belli L, Magistretti G, Puricelli GP, Damiani G, Colombo E and Cornalba GP: Arteritis following intra-arterial chemotherapy for liver tumors. Eur Radiol 7: 323-326, 1997.

9. Lewis AL, Gonzalez MV, Lloyd AW, Hall B, Tang Y, Willis SL, Leppard SW, Wolfenden LC, Palmer RR and Stratford PW: DC bead: In vitro characterization of a drug-delivery device for transarterial chemoembolization. J Vasc Interv Radiol 17: 335-342, 2006.

10. Woo HY and Heo J: Transarterial chemoembolization using drug eluting beads for the treatment of hepatocellular carcinoma: Now and future. Clin Mol Hepatol 21: 344-348, 2015.

11. Lammer J, Malagari K, Vogl T, Pilleul F, Denys A, Watkinson A, Pitton M, Sergent G, Pfammatter T, Terraz S, et al: Prospective randomized study of doxorubicin-eluting-bead embolization in the treatment of hepatocellular carcinoma: Results of the PRECISION V study. Cardiovasc Intervent Radiol 33: 41-52, 2010.

12. Malagari K, Chatzimichael K, Alexopoulou E, Kelekis A, Hall B, Dourakis S, Delis S, Gouliamos A and Kelekis D: Transarterial chemoembolization of unresectable hepatocellular carcinoma with drug eluting beads: Results of an open-label study of 62 patients. Cardiovasc Intervent Radiol 31: 269-280, 2008.

13. Varela M, Real MI, Burrel M,Forner A, Sala M, Brunet M, Ayuso C, Castells L, Montañá X, Llovet JM and Bruix J: Chemoembolization of hepatocellular carcinoma with drug eluting beads: Efficacy and doxorubicin pharmacokinetics. J Hepatol 46: 474-481, 2007.

14. Acunaş B and Rozanes I: Hepatocellular carcinoma: Treatment with transcatheter arterial chemoembolization. Eur J Radiol 32: 86-89, 1999.

15. Malagari K, Pomoni M, Spyridopoulos TN, Moschouris H, Kelekis A, Dourakis S, Alexopoulou E, Koskinas J, Angelopoulos M, Kornezos J, et al: Safety profile of sequential transcatheter chemoembolization with DC Bead ${ }^{\mathrm{TM}}$ : Results of 237 hepatocellular carcinoma (HCC) patients. Cardiovasc Intervent Radiol 34: 774-785, 2011.

16. Poon RT, Tso WK, Pang RW, Ng KK, Woo R, Tai KS and Fan ST: A phase I/II trial of chemoembolization for hepatocellular carcinoma using a novel intra-arterial drug-eluting bead. Clin Gastroenterol Hepatol 5: 1100-1108, 2007.

17. Song DS, Choi JY, Yoo SH, Kim HY, Song MJ, Bae SH, Yoon SK, Chun HJ, Choi BG and Lee HG: DC Bead transarterial chemoembolization is effective in hepatocellular carcinoma refractory to conventional transarterial chemoembolization: A pilot study. Gut Liver 7: 89-95, 2013.

18. Monier A, Guiu B, Duran R, Aho S, Bize P, Deltenre P, Dunet V and Denys A: Liver and biliary damages following transarterial chemoembolization of hepatocellular carcinoma: Comparison between drug-eluting beads and lipiodol emulsion. Eur Radiol 27: 1431-1439, 2017.

19. Toro A, Bertino G, Arcerito MC, Mannnino M, Ardiri A, Patane D and Di Carlo I: A lethal complication after transarterial chemoembolization with drug-eluting beads for hepatocellular carcinoma. Case Rep Surg 2015: 873601, 2015.

20. Bhagat N, Reyes DK, Lin M, Kamel I, Pawlik TM, Frangakis C and Geschwind JF: Phase II study of chemoembolization with drug-eluting beads in patients with hepatic neuroendocrine metastases: High incidence of biliary injury. Cardiovasc Intervent Radiol 36: 449-459, 2013.

21. Guiu B, Deschamps F, Aho S, Munck F, Dromain C, Boige V, Malka D, Leboulleux S, Ducreux M, Schlumberger M, Baudin E and de Baere T: Liver/biliary injuries following chemoembolisation of endocrine tumours and hepatocellular carcinoma: Lipiodol vs. drug-eluting beads. J Hepatol 56: 609-617, 2012.

22. López-Benítez R, Radeleff BA, Barragán-Campos HM, Noeldge G, Grenacher L, Richter GM, Sauer P, Buchler M, Kauffmann G and Hallscheidt PJ: Acute pancreatitis after embolization of liver tumors: frequency and associated risk factors. Pancreatology 7: 53-62, 2007.

23. Khan KN, Nakata K, Shima M, Kusumoto Y, Ishii N, Koji T and Nagataki S: Pancreatic tissue damage by transcatheter arterial embolization for hepatoma. Dig Dis Sci 38: 65-70, 1993.

24. Lewis AL, Taylor RR, Hall B, Gonzalez MV, Willis SL and Stratford PW: Pharmacokinetic and safety study of doxorubicin-eluting beads in a porcine model of hepatic arterial embolization. J Vasc Interv Radiol 17: 1335-1343, 2006.

25. Hong K, Khwaja A, Liapi E, Torbenson MS, Georgiades CS and Geschwind JF: New intra-arterial drug delivery system for the treatment of liver cancer: Preclinical assessment in a rabbit model of liver cancer. Clin Cancer Res 12: 2563-2567, 2006.

This work is licensed under a Creative Commons Attribution-NonCommercial-NoDerivatives 4.0 International (CC BY-NC-ND 4.0) License. 